\title{
Balkanologie
}

Balkanologie Revue d'études pluridisciplinaires

Vol. XIV, $n^{\circ}$ 1-2 | 2012

Volume XIV Numéro 1-2

\section{Le communisme modèle de religion séculière : la Yougoslavie titiste entre héritage stalinien et création originale}

\section{Laëtitia Delamare}

\section{(2) OpenEdition \\ Journals \\ Édition électronique \\ URL : http://journals.openedition.org/balkanologie/2374 \\ DOI : 10.4000/balkanologie.2374 \\ ISSN : 1965-0582 \\ Éditeur \\ Association française d'études sur les Balkans (Afebalk)}

Référence électronique

Laëtitia Delamare, « Le communisme modèle de religion séculière : la Yougoslavie titiste entre héritage stalinien et création originale », Balkanologie [En ligne], Vol. XIV, n 1-2 | 2012, mis en ligne le 08 février 2013, consulté le 17 décembre 2020. URL : http://journals.openedition.org/balkanologie/2374 ; DOI : https://doi.org/10.4000/balkanologie.2374

Ce document a été généré automatiquement le 17 décembre 2020.

(c) Tous droits réservés 


\title{
Le communisme modèle de religion séculière : la Yougoslavie titiste entre héritage stalinien et création originale
}

\author{
Laëtitia Delamare
}

1 L'URSS (Union des Républiques socialistes soviétiques) n'a pas jamais véritablement appliqué le modèle mis en place par la théorie marxiste. La relecture qu'en a fait Lénine, rendue nécessaire par le peu d'indications laissées par Marx et Engels sur la marche à suivre, a conduit à une reformulation qui, si elle sauve le langage et l'option révolutionnaire, en altèrent de nombreux points essentiels. Ainsi le matérialisme historique est-il contesté au nom des libertés éthiques; de même, le primat de l'économique sur le politique et celui de la nécessité historique sur la liberté des sociétés humaines sont remis en question. La mise en place de nouveaux principes, cette fois marxistes-léninistes, vont générer des conséquences imprévues.

2 La plus marquantes d'entre elles est le retour du politique. En effet, Marx considérait cet élément comme faisant partie de la superstructure et, à ce titre, relevant de la domination bourgeoise de même que le droit. À l'inverse, le régime qui s'installe en URSS va le réhabiliter et le projeter au premier plan de son système, notamment par le biais du Parti. Se met alors en place un modèle particulier d'organisation du pouvoir : la «partitocratie idéocratique ». L'expression la plus évidente de ce modèle est la détention légitime du pouvoir par les personnes qui ont en charge l'existence du Parti. Mais ce modèle présente d'autres aspects fondamentaux, qui engendrent l'exacerbation des principes mis en place par Lénine : l'absence d'enracinement du pouvoir dans une base sociologique donnée, l'aliénation sacrificielle au Parti, aspect probant de la religiosité du système étayée par le culte du chef, et enfin la substitution du Parti au peuple.

3 D'autre part, l'évolution du modèle socialiste a été davantage dictée par la résolution de problèmes nés de la mise en place de la collectivisation et de l'industrialisation que 
d'un attachement à une ligne préétablie (notamment dans la seconde moitié des années 30). Cette altération par rapport au modèle marxiste va avoir pour conséquence la reconduction d'un modèle inattendu. Si la profession de l'athéisme est officielle, il n'en demeure pas moins que, cherchant à concilier des éléments nouveaux et anciens (notamment dans le domaine agricole) incompatibles, les Soviétiques composent un modèle contradictoire. En reconduisant la forme religieuse de l'Un tout en la nourrissant d'éléments modernes, ils tentent de concilier l'inconciliable.

4 Si la Yougoslavie de Tito se démarquera des pays frères par la mise en place de structures originales (non-alignement, autogestion), elle reste jusqu'à la rupture avec Staline (1948), voire jusqu'en 1949, un pur produit du Kominterm. Dans cette perspective, elle suit le modèle stalinien, notamment dans la mise en place de la Constitution de 1946 qui se fonde sur la Constitution soviétique de 1936. De manière plus générale, si l'on retient les critères d'encadrement politique, d'idéologisation de l'ensemble des activités sociales et de mobilisation des acteurs autour des buts assignés par la collectivité, la Yougoslavie fait partie des régimes totalitaires nés de la Seconde Guerre mondiale. Dans cette période d'avènement de la Guerre froide, la Yougoslavie est, par son choix d'un modèle d'économie socialiste, partie prenante du bloc de l'Est.

5 Si l'on trouve de nombreux points communs aux deux pays, que ce soit dans les objectifs fixés par le pouvoir (fédéralisme, politique volontariste...) ou dans les conditions objectives (structure sociale, pluralité des nationalités...1), doit-on pour autant considérer que la Yougoslavie titiste n'est qu'un calque de l'URSS stalinienne? Cette question prend une expression singulière dans la devise nationale choisie par Tito: Bratstvo $i$ jedinstvo - «fraternité et unité». Paradoxalement, elle renvoie sémantiquement aux idéaux religieux de lien entre les hommes et de manière plus large à la fonction unificatrice que revêtait la religion dans les sociétés d'ancien régime. En effet, la religion était alors ce qui, via la personne du monarque, permettait de lier le peuple à l'invisible et d'assurer ainsi la cohésion de la société.

6 Les Yougoslaves ont-ils reconduit le modèle religieux de l'Un en imitant les Soviétiques $^{2}$ ? Ont-ils vu dans l'organisation socialiste et dans la mise en place d'un État totalitaire le moyen de répondre à la question de l'unité de la société ? Pour répondre à cette question nous devons d'étudier ce qui, dans la génération de l'Etat yougoslave, relève d'une imitation pure du modèle soviétique, ce qui est création originale et opérer dans ce cas une dichotomie entre les originalités nées d'une volonté propre et celles nécessitées par les aspects particuliers du pays.

7 Si la Yougoslavie se calque, dans un premier temps, parfaitement sur l'URSS tant d'un point de vue théorique que dans les mesures de construction de l'Etat, c'est que les deux pays présentent de grandes convergences d'un point de vue objectif et la Yougoslavie va dans un premier imiter le grand frère soviétique pour répondre à ces problèmes. Cependant, de par ses caractéristiques propres et plus encore suite à la rupture de 1948, la Yougoslavie crée un modèle original qui s'éloigne de l'héritage stalinien.

1.

\section{URSS et Yougoslavie : un même modèle politique}

8 La Première Yougoslavie de Tito (1945-1949) se donne pour modèle l'URSS de Staline. En témoigne la Constitution de 1946 qui renvoie à la Constitution soviétique de 1936 : les fondements de la toute jeune République populaire sont identiques à ceux du grand 
frère soviétique. Nous développerons cette comparaison sur trois points essentiels : l'importance accordée au politique, l'ampleur du rôle du Parti et enfin la mise en place d'une figure tutélaire, en l'occurrence celle du libérateur de la nation : Tito.

1. 1.

\section{Le retour du politique}

9 La Yougoslavie met en place au lendemain de la guerre une surpolitisation de tous les éléments de la vie quotidienne; en particulier, les délits de droit commun se voient revêtus d'une signification politique. Le droit et la loi sont soumis au pouvoir politique, l'adhésion à l'idéologie devient le biais de recrutement politique et économique principal, les fonctions importantes ne sont pas réparties en fonction des mérites et des compétences mais en fonction de critères politiques : tout d'abord la participation à la guerre, l'esprit de Parti (partivnost) et enfin la sympathie à l'égard du communisme.

En fidèle héritier du marxisme-léninisme de type stalinien, le système yougoslave adopte la compatibilité idéologique comme critère de sélection. Reprenant les fondements léninistes de la théorie révolutionnaire, l'activité politique est considérée comme l'apanage d'un groupe de gens formés, d'une élite révolutionnaire. Dès le lendemain de la guerre le PCY (Parti communiste yougoslave) met en place des centres de formation aux échelons local, républicain et fédéral pour former les membres et les cadres du Parti.

11 La politisation de la société s'exprime également de manière exacerbée dans les manuels scolaires qui insistent sur quatre éléments : tout d'abord l'histoire de la guerre de libération nationale, l'histoire de la fondation et de l'évolution du PCY ; l'histoire de l'URSS tient également une place privilégiée; enfin, l'étude théorique regroupe les grands textes du marxisme-léninisme ainsi que celles des théoriciens yougoslaves.

Dans cette perspective, la Yougoslavie se calque sur le modèle totalitaire stalinien de «partitocratie idéocratique » c'est-à-dire la mainmise du Parti sur le pouvoir au nom d'un idéal. La vie des individus est tout entière encadrée par le politique (études, travail, loisirs). L'ensemble des activités est idéologisée et enfin on mobilise la population autour des buts assignés par la collectivité. Ainsi la Yougoslavie, dans son effort de reconstruction et de modernisation, donne-t-elle un aspect idéologique aux grands travaux : la construction de la ligne de chemin de fer Šamac-Sarajevo fut étayée par une propagande très active qui soulignait que c'était un « combat pour le peuple et le développement». On encourage, à l'instar des stakhanovistes soviétiques, les «travailleurs de choc». L'exaltation des travailleurs se fait avec la valorisation du volontarisme auquel renvoie le fameux slogan de la « foi dans le peuple».

13 La Yougoslavie titiste concrétise l'idéologie selon un triple rapport temporel: elle définit une grille de lecture spécifique de l'histoire, introduit des mythes fondateurs et dicte l'attitude à adopter dans l'ensemble des activités humaines en fonction d'un but qu'elle détermine.

1. 1.

\section{Le rôle du Parti}

Le Parti sera le vecteur de l'expression de l'idéologie socialiste. Ses institutions sont de véritables doublons de celles de l'État en Yougoslavie comme en URSS et ce à tous les échelons. La politique du Parti unique permet de souligner le retour du politique : le Parti n'a pas de véritable enracinement sociologique, c'est la superstructure qui 
gouverne. Selon Lénine, le pivot de la légitimation n'est pas affaire de classe mais de conscience de classe. La Yougoslavie se doit de fonder la légitimité d'un pouvoir qui n'a pas d'enracinement sociologique dans un pays à majorité paysanne. Si le mouvement des soviets avait pu légitimer la toute-puissance du Parti en URSS, ce sont la victoire des Partisans, qui apparaît comme le mythe fondateur de la légitimité du Parti communiste, et la figure emblématique de Tito qui joueront ce rôle en Yougoslavie.

Cette politisation de la vie quotidienne est également renforcée, comme en URSS, par l'exaltation du volontarisme. C'est une des évolutions qu'a insufflées Lénine au marxisme en lui donnant un aspect réaliste : la théorie révolutionnaire a vocation à s'incarner dans la réalité mais n'en procède pas nécessairement; c'est le Parti et les forces qu'il permet de mobiliser qui vont permettre de combler l'écart. L'appel aux forces vives du pays se fait sous couvert d'esprit sacrificiel vis-à-vis du Parti car celui-ci est la Révolution en acte. Le volontarisme est alors une religion en soi car il permet de dépasser la réalité tout en la modifiant. Dès lors la vie de la société est mue par l'idéal à atteindre et par l'idéologie. Le Parti met de fait l'État sous tutelle.

Cette mise sous tutelle est double. L'article 14 de la Constitution de 1946 précise que les moyens de production du pays sont la propriété du peuple yougoslave. De fait l'État, donc en dernier ressort le Parti, met cette propriété sous tutelle. Dans un premier temps, il existe en Yougoslavie une confusion entre propriété sociale et propriété étatique. La complexification du processus d'appropriation tient à la personnalisation du pouvoir et de l'État que Tito, auréolé du prestige de la libération, met en place.

17 Le Parti agit donc comme un tampon entre le pouvoir qu'est censé détenir l'État et la mainmise que Tito exerce sur le pouvoir.

1. 1.

\section{La figure tutélaire}

18 L'imitation du modèle soviétique stalinien passe aussi par la mise en place d'un culte du chef. Si Tito est avant tout, comme Staline, l'homme du Parti, son charisme lui donne une dimension qui le distingue quelque peu de son homologue soviétique. On retrouve chez l'un comme chez l'autre un caractère sacré même si l'on devrait plus parler de surhumanité que de divinité du chef. Cependant, les caractères traditionnels attribués à une divinité se retrouvent dans le culte des deux hommes d'État: on leur attribue en effet omniprésence, omnipotence et infaillibilité.

19 La mise en place du culte de Tito $^{3}$ se fait progressivement, mettant en avant tour à tour la figure du héros national empreint de merveilleux et celle du dirigeant de basse extraction proche du peuple. Si le culte de Tito se teindra sensiblement de divin, ce dernier est dès le commencement la figure tutélaire de la Yougoslavie d'après-guerre, les grandes étapes de la vie du pays étant associées à celles de sa vie.

20 La Yougoslavie présente l'originalité dans les premières années de sa vie de vouer également un culte à Staline. Son anniversaire est fêté chaque année dans les grands quotidiens nationaux, il est considéré comme l'exemple à suivre. Il ne faut pas oublier que Tito est un pur produit du Kominterm et que le schisme n'adviendra qu'en 1948, avant cela la Yougoslavie est avant tout un bon élève du grand frère soviétique.

21 Si pour Staline le peuple russe a "besoin d'un tsar ", Tito se donnera plutôt le rôle de clé de voûte de l'édifice yougoslave. En habile tacticien, il crée un rapport tel entre les différentes composantes nationales, religieuses et sociales qu'il devient l'arbitre entre les différentes parties qui pourraient s'affronter au sein de la Fédération. C'est en tant 
que tel qu'il prend une place spécifique qui deviendra divine comme le montre la ritualisation croissante de la fête du 25 mai, anniversaire de Tito.

Dans la mise en place et l'organisation du pouvoir, la Yougoslavie titiste reprend le modèle soviétique et hérite de la forme religieuse de l'Un mise en forme par celle-ci. Trois critères essentiels nous permettent de reconnaître cette forme au-delà de la volonté de modernité et d'athéisme du tout jeune pouvoir socialiste : la politisation de l'ensemble des activités (qui permet une homogénéisation de la vie sociale), la place du Parti qui se substitue au Peuple en le représentant, et enfin la mise en place d'un culte de la personnalité qui assoit le caractère sinon divin du moins surhumain du chef.

1.

\section{Des contraintes communes}

Si l'URSS de Staline a pu servir de modèle à la Yougoslavie titiste dans sa première phase, c'est qu'au-delà du paradigme socialiste, elle avait été confrontée aux mêmes problèmes que la Yougoslavie : importance de la religion, plurinationalité et prégnance de la composante paysanne au sein de la population.

1. 1.

\section{La question religieuse}

Si la Yougoslavie ne présente pas la même homogénéité que l'URSS sur le plan de la foi, il n'en demeure pas moins que nous nous trouvons face à deux pays où l'héritage religieux est particulièrement prégnant. Le système des millets dans l'Empire ottoman a favorisé l'amalgame des fonctions politiques et religieuses et installé une confusion entre appartenance religieuse et nationale. En effet, les interlocuteurs politiques des différents peuples de l'Empire ottoman étaient les chefs de communautés religieuses. Par ailleurs la conversionà l'islam y était synonyme de changement de nationalité mais également la condition pour accéder aux classes sociales privilégiées. La nationalité bosniaque est l'héritière de cette conception de la religion comme marqueur d'identité non seulement culturelle mais également sociale et nationale. Dans un pays où l'émergence des consciences nationales au sein de deux empires s'est faite tardivement, l'impact de l'héritage religieux est extrêmement fort, comme dans la société russe, parce qu'il est marqueur d'identité culturelle voire plus.

En Yougoslavie, comme en URSS, l'héritage religieux prédispose à la reconduction de la forme religieuse même si le contenu en est modifié. Par ailleurs le caractère plurinational, plus prégnant encore en Yougoslavie, a permis la retranscription du modèle soviétique.

1. 1.

\section{La question nationale}

Staline, spécialiste de la question des nationalités, offre à la Yougoslavie l'image de l'homme qui a réussi à la résoudre. La Constitution yougoslave de 1946, malgré le choix du modèle fédéral, laisse en suspens la question essentielle de la visée programmatique du nouvel État: maintien des différentes nationalités comme le laisse entendre le choix de la forme fédérale, ou volonté de créer une citoyenneté spécifiquement yougoslave à l'instar de l'homo sovieticus? Selon Staline, il fallait garder provisoirement une «forme nationale " pour " un contenu socialiste ", le phénomène des nations étant un fait transitoire appelé à disparaître. La position de Tito n'est pas aussi tranchée. 

passera de 31 en 1945 à 1680 en 1949. Comme dans l'URSS des années 20 la situation est difficile : la guerre a ravagé les campagnes yougoslaves (destruction de $50 \%$ du cheptel, politique de la terre brûlée...) et les paysans doivent fournir un effort considérable pour remettre l'agriculture sur pied. La mise en place de la collectivisation dans un climat de violence politique caractérisée mène à une montée de l'opposition paysanne et à une solution de plus en plus incertaine du problème. Le régime hésite alors entre ligne boukharinienne et stalinienne. Il optera finalement pour cette dernière en mettant en place un communisme de guerre et une collectivisation forcée. Pour justifier la répression, la propagande tente de mettre en place la vision d'un koulak riche, mais les grands propriétaires terriens sont rares en Yougoslavie. La répression sociale va alors s'exercer sous forme de remembrement, d'expropriation, d'industrialisation et d'ouvriérisation du paysan. 

nombreux autres domaines, suivi une ligne strictement stalinienne. L'interprétation du marxisme s'est faite, là encore de manière originale, dans la recherche de solutions à des problèmes matériels.

C'est l'un des points communs majeurs qui émergent lors de la comparaison entre URSS et Yougoslavie: tout comme le bolchevisme s'est construit au fur et à mesure, se découvrant à lui-même en fonction de certaines données objectives, le titisme deviendra de plus en plus, en particulier après la rupture avec Staline, un bricolage politique que favorise la mise en place en 1946 d'une Constitution-programme, c'est-àdire ne donnant pas essentiellement la base des institutions mais le but que se propose le tout nouvel État populaire.

1.

\section{La Yougoslavie post-stalinienne : le temps de l'émancipation}

36

Malgré tous les rapprochements que nous avons pu opérer entre l'URSS stalinienne et la première Yougoslavie titiste, notamment dans la reconstruction de la forme religieuse de l'Un, il n'en demeure pas moins que la Yougoslavie présente certaines originalités. Au risque de rappeler l'évidence, la différence essentielle est que la Yougoslavie a un modèle réel. Elle n'a pas à se fonder sur la seule relecture de Marx par Lénine, la preuve de la possibilité d'établir le socialisme ayant déjà été faite. Si les créations les plus connues de la Yougoslavie sont l'autogestion et le non-alignement, nous nous cantonnerons à étudier ici les innovations qu'elle a réalisées dans le domaine des mythes fondateurs, des règlements de la question paysanne et de la question nationale, puisque c'est dans ces domaines que nous avons établi notre comparaison avec l'URSS.

1. 1.

\section{Le mythe fondateur : la guerre de libération nationale}

Si la Russie a pour mythe fondateur la Révolution, l'émergence du socialisme en Yougoslavie a pour contexte la guerre de libération nationale face à l'ennemi fasciste. Si l'on a assisté à une guerre civile dans un premier temps entre tchetniks et oustachis, s'y ajoute bientôt celle entre tchetniks et partisans. L'unité qui émerge après l'épuration de l'immédiat après-guerre, particulièrement importante en Croatie face aux oustachis, repose sur une lutte commune contre l'ennemi extérieur. La Yougoslavie, dépecée lors de la Seconde Guerre mondiale par l'Allemagne, l'Italie et la Bulgarie, trouve son unité dans la lutte contre l'occupant et ses collaborateurs et dans la figure tutélaire de Tito, chef des Partisans.

Durant la période stalinienne du régime titiste, ce mythe fondateur, même s'il est original par rapport au modèle soviétique, permet lui aussi la reconduction du modèle religieux de l'Un. Fort de la multiplicité nationale des partisans et de l'union sacrée des peuples de Yougoslavie face à l'ennemi extérieur, Tito permet de fonder l'unité de son pays sur son image de chef du mouvement de libération.

Si le mythe fondateur diffère entre les deux pays, il engendrera les mêmes conséquences. Fonder l'unité sur une lutte commune, que ce soit contre un ennemi intérieur (les Blancs en Russie) ou extérieur (les Forces de l'Axe en Yougoslavie) : la tentation est grande, lorsque l'unité se délite, de trouver un ennemi commun afin de la

Balkanologie, Vol. XIV, n 1-2 | 2012 
restaurer. Celui-ci peut être extérieur tout comme intérieur au Parti et/ou au pays. Se développe alors une théorie du complot qui sur le long terme altère l'unité en distillant la suspicion dans tous les rapports sociaux. Cependant, il est intéressant de noter que le rayonnement du chef n'est pas pour autant remis en question et que le peuple soupçonne de dysfonctionnement davantage les rouages que l'autorité suprême. La forme de l'Un est sauvegardée grâce à la figure tutélaire du chef.

1. 1.

\section{La résolution de la question paysanne}

La Yougoslavie titiste poststalienne (après 1949 et surtout 1953) résout de manière originale la question paysanne. Plutôt que de mettre en place une prolétarisation de la population paysanne, le régime favorise une ouvriérisation de celle-ci ${ }^{4}$. Le temps de travail est partagé entre le travail à l'usine et le travail passé aux champs, que ce soit pour un individu particulier ou au sein d'une famille selon le sexe ou la génération. Cette option semble assez particulière dans un pays d'obédience marxiste, car il ne permet pas l'émergence d'une conscience de classe à proprement parler.

41 En cela la Yougoslavie se démarque clairement du grand frère soviétique et ce, dès 1952 et surtout après $1953^{5}$. Par la dilution de l'identité paysanne, Tito crée une catégorie hybride qui va permettre en creux un renforcement du pouvoir du Parti. En effet, dans le cadre de l'industrialisation forcée et de la décollectivisation des terres, la polyactivité des travailleurs ne permet pas la naissance de la relation affective au travail que l'on peut trouver au sein de certains métiers industriels, particulièrement dans les branches les plus dures (mine, sidérurgie...). L'émergence de revendications et de syndicats nés d'une solidarité au sein d'un même corps se fait plus difficile lorsque le travail exercé dans le secteur secondaire ne revêt qu'un aspect financier et que le véritable lien affectif se fait par rapport à la terre et au village d'origine. L'investissement politique ne se présente pas alors sous la forme d'une alternative entre organisation syndicale et Parti, celui-ci est le seul lieu d'investissement possible.

Nous sommes en droit de nous demander si cette démarche d'ouvriérisation de la masse paysanne a sciemment été mise en place par Tito pour créer une absence de conscience de classe par l'absence simple de classe, l'engagement politique devant alors nécessairement passer par le Parti, voire l'inexistence d'engagement politique actif et la délégation au Parti du choix des modalités de la vie sociale. On assiste alors au renforcement de la forme de l'Un par la dilution des particularismes sociaux. L'unité ne peut pas se faire à un niveau inférieur à l'État, au Parti et à la figure du chef, et surtout pas contre eux. La solidarité ne se trouve pas entre camarades d'une même équipe mais dans l'effort pour le chef, pour la collectivité, c'est-à-dire non pas pour une réalité palpable mais pour un idéal (nous revenons alors au modèle de la «partitocratie idéocratique »). Cela explique sans doute que le modèle principal d'identification lors du déclin de la Yougoslavie ne sera pas social mais national. Si Tito a laissé en suspens cette question dans la Constitution de 1946, il va, par ses options en politique extérieure, mettre au premier plan les revendications des différentes composantes nationales de la Yougoslavie.

1. 1.

\section{Le patriotisme yougoslave : le tout et les parties}

43 L'approche titiste de la question nationale se fait de manière double et au premier abord paradoxale. D'une part, Tito se fait le défenseur des irrédentismes locaux, 
s'opposant à la Grèce, à l'Autriche et à l'Italie. Il renforce ainsi les particularismes nationaux mais en les incorporant à un patriotisme plus vaste. D'autre part, il exalte et ce avant même la rupture avec Staline - un patriotisme à proprement parler yougoslave. Le passé socialiste du pays est mis en avant. Tito quitte alors son rôle de bon élève de l'URSS, de produit du Kominterm, pour mettre en place un socialisme qui quitte la ligne directrice de l'internationalisme pour se réfugier dans un patriotisme yougoslave se nourrissant des revendications locales.

Cependant le fédéralisme yougoslave, communiste et unitariste, machine à fabriquer de la citoyenneté yougoslave, va connaître une évolution qui l'éloignera de la constitution soviétique de 1936 (par laquelle Staline a dépassé la Constitution de 1924 qui instituait une communauté de nations en employant la manière forte, notamment dans le Caucase). En effet, la reconnaissance de la nation musulmane puis la décentralisation opérée par la Constitution de 1974 mèneront à un renforcement des pouvoirs de décision su niveau républicain tant sur le plan économique que politique.

La tentative titiste d'établir un patriotisme proprement yougoslave sera donc endiguée de part et d'autre par la reconnaissance croissante des nationalités qui composent la Yougoslavie et par l'implication du régime dans le mouvement des non-alignés qui n'est que la suite logique de cette volonté d'originalité titiste. Au finale, la volonté titiste de se démarquer de l'URSS devenue prison des peuples mène à une décentralisation de plus en plus forte qui sera un des ferments de sa désagrégation.

Sur le mythe fondateur de l'Etat et le règlement de la question paysanne, la bifurcation empruntée par le régime yougoslave par rapport au modèle soviétique n'a pas remis en question la reconduction du modèle religieux de l'Un. Cependant, dans le traitement de la question des nationalités et du patriotisme, les options choisies par Tito, qui prendront toute leur envergure dans les années 70 , vont faire éclater cette forme de l'Un en renvoyant au nationalisme comme seule alternative au modèle en place. Ceci est renforcé par l'infaillibilité du chef et le refus catégorique de mettre en place un critère de compétence au lieu de celui de compatibilité idéologique pour recruter les futures élites.

1.

\section{Conclusion}

Par son imitation du modèle stalinien, la Yougoslavie titiste a reconduit le modèle religieux de la forme de l'Un, notamment sous trois aspects particuliers : la politisation de la société dans tous les domaines de la vie sociale, la toute-puissance du Parti et la mise en place d'un culte de la personnalité. Cette imitation a été rendue possible par la similarité des données objectives que présentaient les deux pays. On trouve en effet dans chacun d'eux tout d'abord une forte empreinte de la religion (orthodoxe en Russie; liée à l'héritage du millet comme marqueur d'identité culturelle dans la partie ex-ottomane de la Yougoslavie), ensuite une difficulté d'adaptation de la théorie marxiste-léniniste à une population majoritairement paysanne, enfin une plurinationalité marquée dans les deux populations. Cependant, la Yougoslavie, en particulier dans sa période poststalinienne, présente également des originalités. La présence d'un modèle de socialisme appliqué lui permet de ne pas avoir à inventer l'ensemble des réformes à appliquer. Alors qu'en URSS le mythe fondateur du régime est la Révolution, c'est la Seconde Guerre mondiale qui a joué ce rôle en Yougoslavie en prenant la couleur d'une guerre patriotique de libération nationale. Dans l'immédiat 
après-guerre, la résolution de la question paysanne a pris en Yougoslavie le visage de l'ouvriérisation et non de la prolétarisation du paysan. Cependant, ces deux originalités ont permis à leur manière d'étayer la reconstruction de la forme religieuse de l'Un. Il n'en existe pas moins une brèche dans ce modèle: la résolution de la question des nationalités. La Constitution de 1946 n'a pas tranché entre État citoyen et communauté de nations. Cette ambiguïté entre reconnaissance des nations constituantes et horizon d'une citoyenneté yougoslave peut être considérée comme un des ferments de déliquescence de la société yougoslave puisqu'elle handicape la mise en place de la forme de l'Un contrairement à l'URSS où la Constitution de 1924, qui posait l'Union soviétique comme communauté de nations, sera remplacée en 1936 par une Constitution à visée centralisatrice.

Si dans son imitation de l'URSS la première Yougoslavie titiste a parfaitement reconduit la forme religieuse de l'Un, comme tous les régimes autoritaires du XXème siècle avant et après elle, dans ses créations originales et surtout dans son approche de la question des nationalités elle met à mal ce paradigme. Peut-on pour autant penser que la Yougoslavie aurait pu mener la même politique centralisatrice que l'URSS et sauvegarder ainsi totalement la forme de l'Un? Les structures nationales yougoslaves et soviétiques, si elles se ressemblent par leur multiplicité, ne revêtent pas les mêmes aspects dans leurs proportions. Si la prédominance de la population russe et l'héritage impérial de la Russie déterminent une signification particulière du fédéralisme soviétique, la donne est complètement différente en Yougoslavie. En effet, le passif qui existe entre les peuples serbe et croate détermine de manière fondamentale la construction du fédéralisme (domination serbe de l'entre-deux-guerres, exactions oustachis de la Seconde Guerre mondiale, divergence des cultures politiques consensuelle chez les Croates et conflictuelle chez les Serbes -, différences culturelles héritées des empires respectivement austro-hongrois et ottoman). Tito élabore celui-ci en fonction du passé expansionniste serbe, en pondérant l'importance de la République serbe par la création des deux provinces autonomes.

En donnant aux Yougoslaves une grille de lecture nationale du champ politique dès 1946 mais encore plus avec la Constitution de 1974, Tito condamne de prime abord l'idéal d'une citoyenneté yougoslave. Les recensements de population qui ont lieu tous les dix ans marquent clairement cet échec de l'émergence d'une citoyenneté yougoslave (1,7\% de la population se déclare yougoslave en $1961,1,3 \%$ en 1971, 5,4\% en 1981 et la catégorie a quasiment disparu en 1991).

L'échec de l'unification de la Yougoslavie renvoie donc aux fondements mêmes de la construction de celle-ci. En reprenant la forme de l'Un essentiellement à travers la figure tutélaire du chef infaillible, comme l'illustre le slogan de fin de règne de Tito: Posle Tita, Tito [Après Tito, Tito], la Yougoslavie n'a pas permis l'émergence d'un modèle qui puisse survivre à la disparition de son mentor. L'inféodation du Parti à Tito et le choix partiellement avoué puis assumé d'une Yougoslavie comme communauté de nations ont mis à mal l'idéal socialiste, tel que Lénine l'avait reformulé. L'implosion de la Yougoslavie dans les années 90 renvoie, plus qu'au dégel de conflits que le socialisme aurait mis entre parenthèses durant 40 ans en empêchant de mener une analyse critique des différends nationaux, aux fondements circonstanciels d'un Etat qui n'a pu (n'a pas voulu?) par la suite réformer ses grilles de lecture du champ politique au risque d'un éclatement lors de la disparition de la figure unificatrice. 


\section{NOTES}

1. Nous entendrons ici le nom nationalité et l'adjectif national comme renvoyant aux narodi et aux narodnosti. Voir à ce sujet Gossiaux (Jean-François), Pouvoirs ethniques dans les Balkans, Paris : PUF, 2002, p. 92. Les narodi, au nombre de cinq puis de six, sont les peuples constitutifs de la Yougoslavie c'est-à-dire ceux auxquels correspondent une République : les Serbes, les Croates, les Musulmans (à partir de 1968), les Slovènes, les Monténégrins et les Macédoniens; les narodnosti correspondent aux nationalités qui ne sont pas d'origine slave mais ont un pays de référence à l'extérieur de la Yougoslavie, par exemple les Allemands, les Albanais, les Hongrois, ou les Roumains.

2. Concernant la théorie de la forme religieuse de l'Un voir Gauchet(Marcel), L'Avènement de la démocratie, t. III. À l'épreuve des totalitarismes, 1914-1974, Paris : Gallimard, 2010.

3. Voir Dukic (Suzana), «Un aspect de le propagande titiste : le culte de Tito dans le quotidien Politika (1945 à $1980 »$, Balkanologie 3/1 (1999).

4. Voir Glamočak (Marina), La transition guerrière yougoslave, Paris : L'Harmattan, 2002, p. 35-36

5. Voir Wilson (Duncan), Tito's Yugoslavia, Cambridge : Cambridge University press, 1979.

\section{RÉSUMÉS}

Reprenant l'analyse de Marcel Gauchet qui assimile les grands courants idéologiques du XXe siècle à des religions séculières, cet article se propose d'analyser la marque de l'héritage de la constitution stalinienne de 1936 dans la constitution yougoslave de 1946. L'analyse porte tout d'abord sur les similitudes entre les deux constitutions dans leur reconduction du modèle de l'Un et les grandes questions auxquelles se trouvent confrontés les deux régimes; la suite de l'article se penche sur les différences que présente la Yougoslavie titiste avec l'URSS qui, conjuguées avec la rupture avec Staline, mèneront à une émancipation de l'idéologie yougoslave.

\section{INDEX}

Mots-clés : Marcel Gauchet, sécularisation, Tito, Yougoslavie

Keywords : Marcel Gauchet, secularization, Tito, Yugoslavia

\section{AUTEUR}

\section{LAËTITIA DELAMARE}

Doctorante au IIAC-LAIOS (CNRS/EHESS) 